\title{
The Upper Bound of Block Number for Group Divisible Nuclear Design with Block Size 4
}

\author{
Yufeng Gao ${ }^{1,2}$ \\ ${ }^{1}$ Institute of Mathematics, Beijing Jiaotong University, Beijing 100044, P. R. China \\ ${ }^{2}$ College of Mathematics, Tonghua Normal University, Tonghua 134000, P. R. China
}

\begin{abstract}
Nuclear design could be used for constructing packing and covering of a graph in combinatorial design theory. We generalize the nuclear design to group divisible nuclear design, and discuss the upper bound of block number for group divisible nuclear design with block size 4 .
\end{abstract}

\section{Keywords—group divisible; nuclear design; packing; covering}

\section{INTRODUCTION}

Nuclear design is an important conception in combinatorial design theory. Let $\mathrm{H}$ be a simple graph and $\mathrm{G}$ be a set of simple graphs. Let $\lambda$ be a positive integer and $\lambda \mathrm{H}$ denote the graph $\mathrm{H}$ with each of its edges replicated $\lambda$ times. Let $X$ be the vertex set of $\mathrm{H}$, we define a $(\lambda \mathrm{H} ; \mathrm{G})$-nuclear design as follows:

(1) A $(\lambda H ; G)$-packing is a pair $(X, A)$ where $A$ is a collection of subgraphs (called blocks) of $\lambda \mathrm{H}$, such that each block is isomorphic to a graph of $\mathrm{G}$, and each edge of $\lambda \mathrm{H}$ is contained in at most $\lambda$ blocks of A . $|\mathrm{A}|$ is as large as possible.

(2) A $(\lambda \mathrm{H} ; \mathrm{G})$-covering is a pair $(X, \mathrm{~B})$ where $\mathrm{B}$ is a collection of subgraphs (called blocks) of $\lambda \mathrm{H}$, such that each block is isomorphic to a graph of $\mathrm{G}$, and each edge of $\lambda \mathrm{H}$ is contained in at least $\lambda$ blocks of $\mathrm{B}$. $|\mathrm{B}|$ is as small as possible.

(3) A $(\lambda \mathrm{H} ; \mathrm{G})$-nuclear design is a pair $(X, \mathrm{C})$ where $\mathrm{C}$ is a collection of subgraphs (called blocks) of $\lambda \mathrm{H}$, such that $\mathrm{C}=\mathrm{A} \cap \mathrm{B}$ and $\mathrm{C}$ is as large as possible among all intersections of any maximum $A$ and minimum $B$.

In this paper, we discuss the situation $\lambda=1$. When $H$ is the complete graph $K_{v}$, a $\left(K_{v} ; \mathrm{G}\right)$-packing is denoted by a

$\mathrm{P}(\mathrm{v} ; \mathrm{G} ; 1)$, a $\left(K_{v} ; \mathrm{G}\right)$-covering is denoted by a $\mathrm{C}(\mathrm{v} ; \mathrm{G} ; 1)$. When $\mathrm{H}$ is the complete multipartite graph $K_{u_{t}, u_{2}, \ldots, u_{t}}$, a $\left(K_{u_{1}, u_{2}, \ldots, u_{t}} ; \mathrm{G}\right)$-packing is said to be a group divisible packing, written as a G-GDP of type $\left\{u_{1}, u_{2}, \ldots, u_{t}\right\}$, a $\left(K_{u_{1}, u_{2}, \ldots, u_{t}} ; \mathrm{G}\right)$ covering is said to be a group divisible covering, written as a G-GDC of type $\left\{u_{1}, u_{2}, \ldots, u_{t}\right\}$. Here the multiset $\left\{u_{1}, u_{2}, \ldots, u_{t}\right\}$ is called the group type (or type) of the GDP or GDC. For simplicity, we use $g_{1}^{n_{1}}, g_{2}^{n_{2}}, \ldots, g_{r}^{n_{r}}$ to denote the $n_{i}$ occurrences of $g_{i}^{n_{i}}$ for $1 \leq i \leq r$ in group types. Obviously, a G-GDP of type $1^{v}$ is just a $\mathrm{P}(\mathrm{v} ; \mathrm{G} ; 1)$ and a G-GDC of type $1^{v}$ is just a $\mathrm{C}(\mathrm{v} ; \mathrm{G} ; 1)$.

Note that, a $\left(K_{u_{1}, u_{2}, \ldots, u_{t}} ; \mathrm{G}\right)$-nuclear design is said to be a Ggroup divisible nuclear design, written as a G-GDND of type $\left\{u_{1}, u_{2}, \ldots, u_{t}\right\}$.

\section{THE UPPER BOUND OF BLOCK NUMBER}

A $(H ; G)$-packing $(X, A)$ is called maximum if there does not exist any $(\mathrm{H} ; \mathrm{G})$-packing $\left(X, \mathrm{~A}^{\prime}\right)$ with $|\mathrm{A}|<\left|\mathrm{A}^{\prime}\right|$. The leave graph (or leave) of a $(\mathrm{H} ; \mathrm{G})$-packing $(X, \mathrm{~A})$ is the graph $(X, \mathrm{~L})$ which is the collection of unused edges. A $(H ; G)$-covering $(X, B)$ is called minimum if there does not exist any $(H ; G)-$ covering $\left(X, \mathrm{~B}^{\prime}\right)$ with $|\mathrm{B}|<\left|B^{\prime}\right|$. The excess graph (or excess) of a $(\mathrm{H} ; \mathrm{G})$-covering $(X, \mathrm{~B})$ is the graph $(X, \mathrm{E})$ which is the collection of edges with multiplicity more than one. If the leave graph of a $(\mathrm{H} ; \mathrm{G})$-packing is null, then such a packing is maximum, and referred to as a $(\mathrm{H} ; \mathrm{G})$-design. We always write G-MGDP instead of maximum G-GDP. The packing number of $D\left(G, g^{n}\right)$ is the number of blocks in a G-MGDP of type $g^{n}$.

In this paper, we concern the situation about $G=K_{3}+\mathrm{e}$ and $G=K_{4}-\mathrm{e} . K_{3}+\mathrm{e}$ is a graph with vertices a,b,c,d and edges ab,bc,ac,cd, denoted by $(a, b, c)-d . \quad K_{4}-$ e is a graph with vertices a,b,c,d and edges ab,ac,ad,bc,bd, denoted by [a,b,c-d].

The upper bound of $D\left(K_{3}+\mathrm{e}, g^{n}\right)$ is as follows.

$$
D\left(K_{3}+\mathrm{e}, g^{n}\right) \leq\left\lfloor n(n-1) g^{2} / 8\right\rfloor
$$

If $D\left(K_{3}+\mathrm{e}, g^{n}\right)$ could attain $\left\lfloor n(n-1) g^{2} / 8\right\rfloor$, then number of edges in the leave would be

$$
\frac{n(n-1) g^{2}}{2}-4\left\lfloor\frac{n(n-1) g^{2}}{8}\right\rfloor= \begin{cases}0, & n(n-1) g^{2} \equiv 0,1(\bmod 8), \\ 1, & n(n-1) g^{2} \equiv 2,7(\bmod 8), \\ 2, & n(n-1) g^{2} \equiv 4,5(\bmod 8), \\ 3, & n(n-1) g^{2} \equiv 3,6(\bmod 8)\end{cases}
$$

Then the possible leave would be 
TABLE I. POSSIBLE LEAVES AMONG (K3+e)-MGDP OF TYPE $\mathrm{g}{ }^{n}$

\begin{tabular}{c|c|cccc}
\hline \multicolumn{2}{c|}{$\mathrm{n}$} & \multicolumn{4}{c}{$\mathrm{n}(\bmod 8)$} \\
\cline { 3 - 6 } $\mathrm{g}$ & 0,1 & 2,7 & 3,6 & 4,5 \\
\hline \multirow{2}{*}{$\mathrm{g}(\bmod 2)$} & 0 & $\varnothing$ & $\varnothing$ & $\varnothing$ & $\varnothing$ \\
\cline { 3 - 6 } & 1 & $\varnothing$ & E1 & E3 & E2 \\
\hline
\end{tabular}

The upper bound of $D\left(K_{4}-\mathrm{e}, g^{n}\right)$ is as follows.

$$
D\left(K_{4}-\mathrm{e}, g^{n}\right) \leq\left\lfloor n(n-1) g^{2} / 10\right\rfloor
$$

If $D\left(K_{4}-\mathrm{e}, g^{n}\right)=\left\lfloor n(n-1) g^{2} / 10\right\rfloor$, then number of edges in the leave would be

$$
\frac{n(n-1) g^{2}}{2}-5\left\lfloor\frac{n(n-1) g^{2}}{10}\right\rfloor= \begin{cases}0, & n(n-1) g^{2} \equiv 0(\bmod 10), \\ 1, & n(n-1) g^{2} \equiv 2(\bmod 10), \\ 2, & n(n-1) g^{2} \equiv 4(\bmod 10), \\ 3, & n(n-1) g^{2} \equiv 6(\bmod 10), \\ 4, & n(n-1) g^{2} \equiv 8(\bmod 10)\end{cases}
$$

Then the possible leave wold be

TABLE II. POSSIBLE LEAVES AMONG (K4-e)-MGDP OF TYPE g ${ }^{n}$

\begin{tabular}{c|c|ccccc}
\hline \multirow{2}{*}{$\begin{array}{l}\mathrm{n} \\
\mathrm{g}\end{array}$} & \multicolumn{5}{c}{$\mathrm{n}(\bmod 5)$} \\
\cline { 3 - 7 } & 0 & 1 & 2 & 3 & 4 \\
\hline \multirow{4}{*}{$\operatorname{g}(\bmod 5)$} & 0 & $\varnothing$ & $\varnothing$ & $\varnothing$ & $\varnothing$ & $\varnothing$ \\
\cline { 2 - 7 } & 1 & $\varnothing$ & $\varnothing$ & $\mathrm{E} 1$ & $\mathrm{E} 3$ & $\mathrm{E} 1$ \\
\cline { 2 - 7 } & 2 & $\varnothing$ & $\varnothing$ & $\mathrm{E} 4$ & $\mathrm{E} 2$ & $\mathrm{E} 4$ \\
\cline { 2 - 7 } & 3 & $\varnothing$ & $\varnothing$ & $\mathrm{E} 4$ & $\mathrm{E} 2$ & $\mathrm{E} 4$ \\
\cline { 2 - 7 } & 4 & $\varnothing$ & $\varnothing$ & $\mathrm{E} 1$ & $\mathrm{E} 3$ & $\mathrm{E} 1$ \\
\hline
\end{tabular}

In table 1 and $2, E_{i}$ is any graph with $i$ edges, for $i=1,2,3,4$.We exhibit all the possible leave graph as follows. For $E_{i, j}, i$ is the edge number and $j$ is the serial number.

We denote $N\left(G, g^{n}\right)$ the number of blocks in a G-GDND of type $g^{n}$. Combine the above discussion and definition, we obtain the following theorem.

Theorem 2.1 When $G=K_{3}+\mathrm{e}$ and $G=K_{4}-\mathrm{e}$, the upper bound of block number for G-GDND of type $\mathrm{g}^{n}$ as follows:

(1) $N\left(K_{3}+\mathrm{e}, g^{n}\right) \leq D\left(K_{3}+\mathrm{e}, g^{n}\right) \leq\left\lfloor n(n-1) g^{2} / 8\right\rfloor$,

(2) $N\left(K_{4}-\mathrm{e}, g^{n}\right) \leq D\left(K_{4}-\mathrm{e}, g^{n}\right) \leq\left\lfloor n(n-1) g^{2} / 10\right\rfloor$.

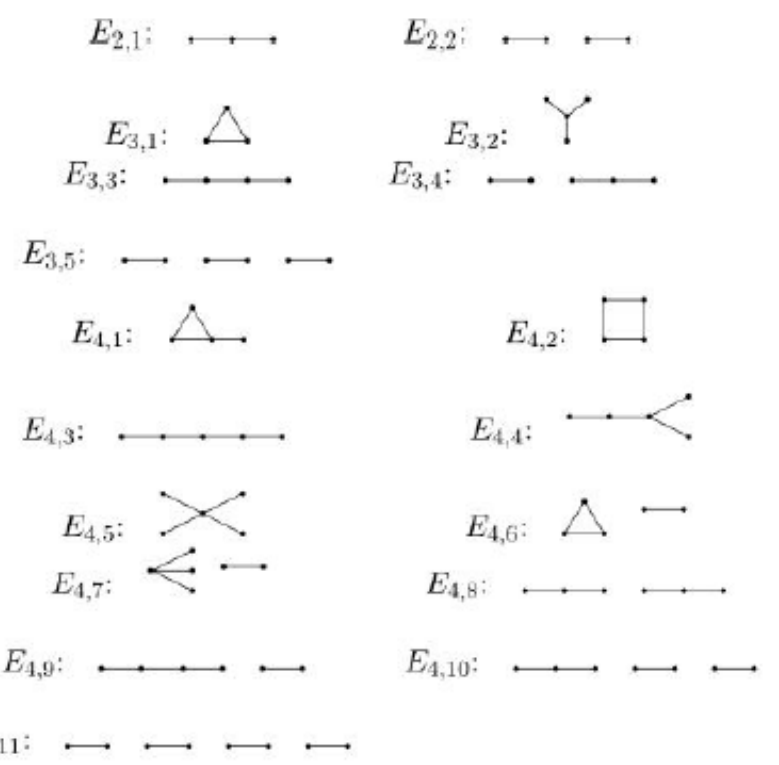

FIGURE I. SIMPLE GRAPHS OF 2 EDGES, 3EDGES AND 4 EDGES

\section{REFERENCES}

[1] Mendelsohn E, Shalaby N, SHEN Hao. Nuclear Designs[J]. Ars Combin, 1991, 32(2) : 225-238.

[2] Billington E J, Lindner C C. Maximum Packings of Uniform Group Divisible Triple Systems[J]. Journal of Combinatorial Designs, 1996, 4(6) : 397-404

[3] HU Xijuan, CHANG Yanxun, FENG Tao. Group Divisible Packings and Coverings with Any Minimum Leave and Minimum Excess[J]. Graphs \& Combinatorics, 2016, 32(4) : 1423-1446.

[4] Fort M K, Hedlund G A. Minimal Coverings of Pairs by Triples[J]. Pacific Journal of Mathematics, 1958, 8(4) : 709-719.

[5] Heinrich K, YIN Jianxing. On Group Divisible Covering Designs[J]. Discrete Mathematics, 1999, 202(1-3) : 101-112.

[6] Colbourn C J, Ling A C H, Quattrocchi G. Minimum Embedding of P3designs into (K4-e)-designs[J]. Journal of Combinatorial Designs, 2003, 11(5) : 352-366.

[7] Hoffman D G, Lindner C C, Sharry M J. Maximum Packings of Kn with Copies of (K4-e) [J]. Aequationes Mathematicae, 1996, 51(3) : 247-269.

[8] Lindner C C, Street A P. Simple Minimum Coverings of Kn with Copies of (K4-e) [J]. Aequationes Mathematicae, 1996, 52(1) : 284-301.

[9] GAO Yufeng, CHANG Yanxun, FENG Tao. Group Divisible (K4-e)packings with Any Minimum Leave[J]. Journal of Combinatorial Designs, 2018, 26(7) : 315-343.

[10] Colbourn C J, Hoffman D G, Rees R, et al. A New Class of Group Divisible Designs with Block Size Three[J]. Journal of Combinatorial Theory, 1992, 59(1): 73-89. 